\title{
Diffuse sclerosing variant of papillary thyroid carcinoma presenting as suspected cervical osseous metastasis: a case report and literature review
}

\author{
Ari Chong', Kweon-cheon $\mathrm{Kim}^{2}$, Yoo-Seok Kim², Ran Hong ${ }^{3}$ \\ Departments of ${ }^{1}$ Nucler Medicine, ${ }^{2}$ Surgery and ${ }^{3}$ Pathology, Chosun University College of Medicine, Gwangju, Korea
}

Received April 2, 2021

Revised April 15, 2021

Accepted April 15, 2021

Corresponding author

Ran Hong

Department of Pathology, Chosun

University College of Medicine, 365

Philmundaero, Dong-gu, Gwangju

61453, Korea

Tel: $+82-62-230-6356$

Fax: +82-62-226-5860

E-mail: nanih@chosun.ac.kr

ORCID:

https://orcid.org/0000-0001-7011-5215
The diffuse sclerosing variant of papillary thyroid carcinoma (DSV-PTC) is an uncommon variant of PTC. Although its histologic features are aggressive, its prognostic significance remains controversial. Herein, we report a case of DSV-PTC, in a 41-year-old woman with a history of breast and thyroid cancers. Bone scan evaluation showed suspected cervical osseous metastasis during the previous cancer follow-up. Further, magnetic resonance imaging was recommended. Preoperative fine-needle aspiration (FNA) showed features of thyroid papillary carcinoma and postoperatively, the mass was diagnosed to DSV-PTC showing features of numerous and diffuse calcification and squamous metaplasia. Herein, we describe the pathologic features, including histologic and FNA cytologic features, and prognostic implications through a literature review.

Keywords: Papillary carcinoma; Sclerosis; Calcification; Lymphatic emboli

\section{INTRODUCTION}

The diffuse sclerosing variant of papillary thyroid carcinoma (DSV-PTC) is a rare variant of PTC, with prevalence of $0.3 \%-5.3 \%$ [1,2]. It was firstly described by Vickery et al. [3] as a variant of PTC in 1985 and was named "DSV-PTC" in the World Health Organization (WHO) classification [4]. It has unique histologic features such as diffuse and dense sclerosis, numerous calcifications including psammoma bodies, squamous metaplasia, and a background of lymphocytic thyroiditis [5]. In addition, it shows exuberant lymphatic invasion, extrathyroidal extension, frequent cervical lymph node metastasis, and distant metastasis, suggesting an aggressive behavior [5]. However, owing to its rarity, the clinicopathologic characteristics and prognostic significance including the probability of disease-specific survival (DSS) and overall survival (OS) have not been well characterized and are therefore debated. In addition, it is associated with a genetic alteration different from conventional PTC (C-PTC). While the BRAF mutation is more common in C-PTC, the $R E T / P T C$ rearrangement is the major genetic alteration in DSV-PTC [5].

Herein, we report a case of DSV-PTC in a 41-year-old woman with a history of breast and thyroid cancers. Postoperatively, she was regularly checked for the detection of tumor recurrence or metastasis through various modalities. 
The bone-scan examination revealed an atypical lesion, which was suspected to be cervical osseous metastasis. Fine needle aspiration (FNA) cytology led to the diagnosis of PTC. Total thyroidectomy of the residual thyroid was performed, and the patient was pathologically diagnosed with DSVPTC. Due to the pathologic features of numerous and diffuse calcifications of DSV-PTC, osseous metastasis was suspected in the bone scan. Herein, we describe the pathologic features, including histologic and FNA cytologic features, and prognostic implications through a literature review.

The study was approved by the ethics committee of Chosun University Hospital (Institutional review Board of Chosun university hospital, Gwangju, Korea), who waived the requirement for written informed consent due to the nature of the study (No. 2020-08-003).

\section{CASE REPORT}

\section{Clinical summary}

A 41-year-old woman with a history of breast and thyroid cancers who had undergone breast-conserving mastectomy and subtotal thyroidectomy in 2014 was detected with a thyroid mass at a regular follow-up. On ultrasonography, the mass measured $1.0 \mathrm{~cm}$ and showed an irregular margin. The ${ }^{99 \mathrm{~m}} \mathrm{Tc}$-hydroxymethylene diphosphonate (HDP) bone scan identified an atypical cervical lesion (Fig. 1). Because

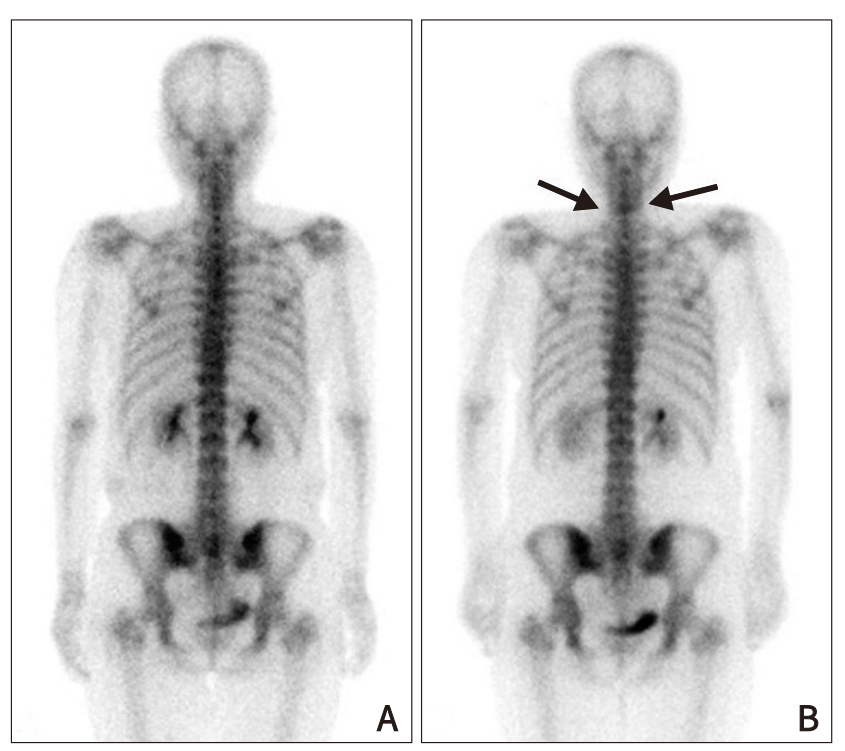

Fig. 1. ${ }^{99 \mathrm{~m}} \mathrm{Tc}$-hydroxymethylene diphosphonate (Tc 99m HDP) bone scan showed increased uptake of radionuclide tracer in cervical area (B, arrows), which lesion was not observed 4 month ago (A). of the young age of the patient, degenerative changes were unlikely. In the nuclear medicine department, the patient was suspected with cervical osseous metastasis. Further, magnetic resonance imaging was recommended for confirmation.

\section{Pathological findings}

For the differential diagnosis of cervical osseous metastasis from a previous cancer and primary thyroid cancer, we performed FNA cytology under ultrasound guidance. Three passes were randomly performed in the left lobes. One smears was prepared from each pass and immediately fixed in $95 \%$ ethanol. Slides were stained with the Papanicolaou (PAP) staining method. Microscopically, several syncytial fragments of medium-sized cells were observed, including papillary fronds, atypical nuclei with the typical features of papillary carcinoma such as nuclear enlargement and pleomorphism, an irregular nuclear membrane, grooves, and scattered intranuclear inclusions. With these typical PTC-like cellular appearances, predominant squamous metaplastic epithelium and abundant psammoma bodies were observed with small lymphocytic infiltration (Fig. 2G). These features were suggestive of the diagnosis of PTC, and a note of caution regarding squamous metaplasia was added. Finally, the patient was diagnosed with the thyroid cancer, and possibility of osseous metastasis was ruled out. Subsequently, residual total thyroidectomy and central neck dissection were performed. Macroscopically, a $2.2 \times 1.8 \mathrm{~cm}-$ sized, whitish-gray hard mass was detected. Microscopically, the lesion filled the entire thyroid, and had an ill-defined border. The lesion comprised the basically classic papillary structures alternating with a more solid sheet of stoma infiltrated with cancer cell nets. Extensive squamous metaplasia was evident within the papillary fronds (Fig. 2A-C). Immunohistochemically, these squamous metaplastic areas were positive for thyroid transcription factor-1 (TTF-1), P40 (Fig. 2D, E), and cytokeratin19 (CK19). The parenchyma showed florid lymphocytic thyroiditis (Fig. 2B, C). A large amount of parenchyma was replaced by dense fibrosis. Frequent calcified areas with numerous psammoma bodies were found within the tumor nests (Fig. 2A-C). Multifocal D2-40 (+) endolymphatic invasions were frequently noted (Fig. 2F). One lymph node was positive for metastasis. The pathological stage was determined to be pT2 N1a. 

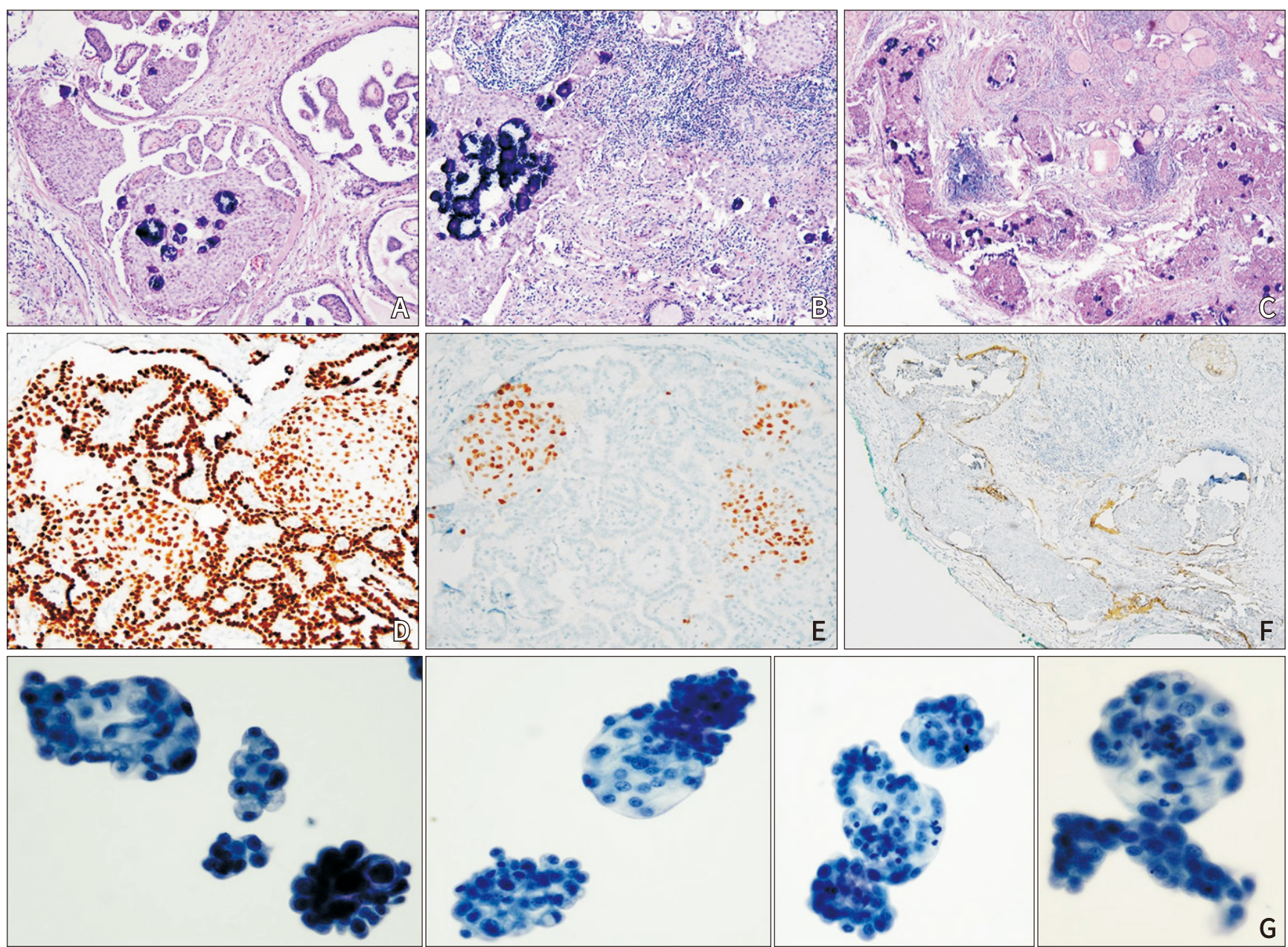

Fig. 2. Microscopic findings of the tumor. In the background of typical papillary thyroid carcinoma, tumor nests showing squamous metaplasia (A) are frequently found, this area is immunoreactive for thyroid transcription factor-1 (TTF-1) (D), P40 (E). Multifocal numerous psammoma bodies (A, B, C) and lymphocytic thyroiditis $(\mathrm{B}, \mathrm{C})$ are also noted. Lymphatic tumor emboli are observed within D2-40 (+) lymphatics (F). In preoperative fine needle aspiration cytology, multiple squamoid nests showing flat, polygonal shape with sharply demarcated cell membrane without keratinization, psammoma bodies and lymphocytic infiltrates are found $(G)$.

\section{DISCUSSION}

The WHO classification of endocrine tumors [5] describes several variants of PTC, including the papillary microcarcinoma, encapsulated variant, follicular variant, DSV, tall cell variant (TCV), cribriform-morular variant, hobnail variant, PTC with fibromatosis/fasciitis-like stroma, solid/trabecular variant, oncocytic variant, spindle cell variant and clear cell variant. DSV-PTC occurs more commonly in women, most frequently in the second or third decade of life [1,6]. The clinical presentation typically includes diffuse enlargement of the thyroid gland. Elevated serum anti-thyroglobulin and anti-microsomal antibodies may mimic Hashimoto's thyroiditis [7]. Histologically, DSV-PTC is characterized by dene sclerosis, numerous psammoma bodies, and background changes of chronic lymphocytic thyroiditis. Tumor nests appear solid, with associated squamous metaplasia, including squamous morules. Tumor cells have a propensity to invade the intrathyroidal lymphatic spaces and tendency to show extrathyroidal extension [5]. Compared to C-PTC, DSVPTC is associated with higher incidences of extrathyroidal extension, cervical lymph node involvement, and distant metastasis, primarily to the lung [8]. However because of its rarity, the clinicopathological characteristics, probability of prognostic significance after thyroidectomy and adjuvant modalities (radioiodine-131 therapy and neck irradiation) are not well characterized. Al-Qahtani et al. [2], in a study of 44 cases of DSV-PTC, suggested that it is more aggressive 
variant compared to C-PTC and shows an inferior DSS rate.

Several studies have reported an aggressive behaviors and a poor prognosis [2,9-11]. Akaishi et al. [0] described that most patients with DSV-PTC were young and had a background of chronic thyroiditis. Outcomes for DSV were good, but recurrence was more common than in classical PTC. Moreover, according to Moreno et al. [12], DSV-PTC has the following features: (a) younger age of presentation (b) greater affinity for men (c) greater frequency of extrathyroidal extension and (d) greater frequency of recurrences. They suggested that the clinical importance of recognizing this variant is based on the need to apply a more aggressive treatment and more exhaustive follow-up for such patients. Kazaure et al. [13] reported that although DSV and TCV are rare, they are increasing in incidence and have a worse prognosis compared to C-PTC. Patients with these variants were treated aggressively with thyroidectomy and radioiodine, regardless of the tumor size in a large-scale study of 43,738 patients. Recently, a systemic review and meta-analysis of DSV-PTC by Vuong et al. [14] concluded that highrisk PTC should be considered because of the high propensities for tumor invasion, metastasis, relapse, and mortality. The aggressiveness of DSV-PTCs might be related to a different molecular pathway than that of C-PTC.

Although it is controversial, in a study of the clinical behavior and prognosis of DSV-PTC and insular carcinoma, Albareda et al. [15] proposed that patients with DSV-PTC do not require a different treatment than that given to C-PTC patients, and in contrast, insular carcinoma cases require a more aggressive therapeutic approach. Lam and Lo [16] also found that the prognosis of DSV-PTC was similar to that of C-PTC. Yamashita et al. [17] reported that the overall mortality and disease-specific mortality rates showed no significant difference between the DSV-PTC and non-DSV-PTC groups; therefore, they proposed that DSV-PTC is not an aggressive subtype of PTC and that patients with DSV-PTC do not require a more aggressive therapy than those with non-DSV-PTC in the same stage. In the WHO classification of Tumors of the endocrine organs, the authors concluded that DSV-PTC is associated with a shorter DFS. However, the mortality rates are comparable to those of C-PTC, possibly due to the favorable effect of young age, with $93 \%$ of the 10-year DSS [5].

Genetic alterations in DSV-PTC are also different from those in C-PTC. While BRAF mutation is the most common mutation in C-PTC, in DSV-PTC, the RET/PTC rearrangement is frequently found in DSV-PTC and BRAF mutations are rare [18-20]. According to Li et al. [21], their meta-analysis of BRAF V600E mutation and its association with clinicopathological features of PTC identified that unlike RET/ PTC rearrangement, BRAF mutations are associated with aggressive clinical manifestations and poor outcomes. This result suggests that the biological aggressiveness of DSVPTCs might be related to a different molecular pathway than the RET/PTC rearrangement. However, Joung et al. [19] analyzed the subtype of the RET/PTC rearrangement. They demonstrated that the RET/PTC rearrangement was the major genetic alteration in patients with DSV-PTC. The RET/PTC1 rearrangement was the most common genetic alteration in DSV-PTC patients and was associated with favorable outcomes. Also, it was associated with an advanced stage at the diagnosis and poor clinical outcomes, including poor response to therapy [19].

The present patient had a history of breast and thyroid cancers. Regular inspection, including radiologic studies, is essential for the cancer follow-up. Calcified/osseous images of the cervical area may suggest an osseous metastatic lesion in the cervical area. Awareness of this category showing marked dystrophic calcification may guide the treatment strategies and prevent misdiagnoses.

Herein, we reported a case of DSV-PTC in a 41-year-old woman with history of breast and thyroid cancers. In the follow-up HDP scan, a cervical mass lesion suggested cervical osseous metastasis. However, FNA cytology identified thyroid papillary carcinoma, and the surgical specimen showed typical microscopic findings of DSV-PTC. DSVPTC is a rare variant of PTC with distinct clinicopathological and molecular characteristics. It is commonly seen in young patients. Although the prognostic behavior is not well characterized, appropriate treatments should be considered because of the higher incidences of lymph node and distant metastasis and high recurrence rates.

\section{ACKNOWLEDGEMENTS}

This study was supported by research funds from Chosun University Hospital, Korea, 2020. 


\section{CONFLICT OF INTEREST}

No potential conflict of interest relevant to this article was reported.

\section{REFERENCES}

1. Soares J, Limbert E, Sobrinho-Simões M. Diffuse sclerosing variant of papillary thyroid carcinoma. A clinicopathologic study of 10 cases. Pathol Res Pract 1989;185:200-6.

2. Al-Qahtani KH, Al Asiri M, Tunio MA, Aljohani NJ, Bayoumi Y, AlShakwer W. Diffuse sclerosing variant papillary thyroid carcinoma: clinicopathological and treatment outcome analysis of 44 cases. Kuwait Med J 2015;47:225-30.

3. Vickery AL Jr, Carcangiu ML, Johannessen JV, Sobrinho-Simoes M. Papillary carcinoma. Semin Diagn Pathol 1985;2:90100.

4. DeLellis RA, Lloyd RV, Heitz PU, Eng C. World Health Organization classification of tumours. Pathology and genetics of tumours of endocrine organs. Lyon: International Agency for Research on Cancer; 2004.

5. Lloyd RV, Osamura RY, Klöppel G, Rosai J. WHO classification of tumours of endocrine organs. 4th ed. Lyon: International Agency for Research on Cancer; 2017.

6. Carcangiu ML, Bianchi S. Diffuse sclerosing variant of papillary thyroid carcinoma. Clinicopathologic study of 15 cases. Am J Surg Pathol 1989;13:1041-9.

7. Vukasović A, Kuna SK, Ostović KT, Prgomet D, Banek T. Diffuse sclerosing variant of thyroid carcinoma presenting as Hashimoto thyroiditis: a case report. Coll Antropol 2012;36 Suppl 2:219-21.

8. Koo JS, Hong S, Park CS. Diffuse sclerosing variant is a major subtype of papillary thyroid carcinoma in the young. Thyroid 2009;19:1225-31.

9. Akaishi J, Sugino K, Kameyama K, Masaki C, Matsuzu K, Suzuki A, et al. Clinicopathologic features and outcomes in patients with diffuse sclerosing variant of papillary thyroid carcinoma. World J Surg 2015;39:1728-35.

10. Falvo L, Giacomelli L, D’Andrea V, Marzullo A, Guerriero G, de Antoni E. Prognostic importance of sclerosing variant in papillary thyroid carcinoma. Am Surg 2006;72:438-44.

11. Regalbuto C, Malandrino P, Tumminia A, Le Moli R, Vigneri $\mathrm{R}$, Pezzino V. A diffuse sclerosing variant of papillary thyroid carcinoma: clinical and pathologic features and outcomes of 34 consecutive cases. Thyroid 2011;21:383-9.

12. Moreno Egea A, Rodriguez Gonzalez JM, Sola Perez J, Soria T, Parrilla Paricio P. Clinicopathological study of the diffuse sclerosing variety of papillary cancer of the thyroid. Presentation of 4 new cases and review of the literature. Eur J Surg Oncol 1994;20:7-11.

13. Kazaure HS, Roman SA, Sosa JA. Aggressive variants of papillary thyroid cancer: incidence, characteristics and predictors of survival among 43,738 patients. Ann Surg Oncol 2012;19:1874-80.

14. Vuong HG, Kondo T, Pham TQ, Oishi N, Mochizuki K, Nakazawa T, et al. Prognostic significance of diffuse sclerosing variant papillary thyroid carcinoma: a systematic review and meta-analysis. Eur J Endocrinol 2017;176(4):433-41.

15. Albareda M, Puig-Domingo M, Wengrowicz S, Soldevila J, Matias-Guiu X, Caballero A, et al. Clinical forms of presentation and evolution of diffuse sclerosing variant of papillary carcinoma and insular variant of follicular carcinoma of the thyroid. Thyroid 1998;8:385-91.

16. Lam AK, Lo CY. Diffuse sclerosing variant of papillary carcinoma of the thyroid: a 35-year comparative study at a single institution. Ann Surg Oncol 2006;13:176-81.

17. Yamashita H, Noguch S, Takahashi H, Watanabe S, Uchino S, Murakami T, et al. The diffuse sclerosing variant of papillary thyroid carcinoma is not an aggressive subtype of papillary carcinoma. J Thyroid Disorders Ther 2014;3:1-4.

18. Lim JY, Hong SW, Lee YS, Kim BW, Park CS, Chang HS, et al. Clinicopathologic implications of the BRAF(V600E) mutation in papillary thyroid cancer: a subgroup analysis of 3130 cases in a single center. Thyroid 2013;23:1423-30.

19. Joung JY, Kim TH, Jeong DJ, Park SM, Cho YY, Jang HW, et al. Diffuse sclerosing variant of papillary thyroid carcinoma: major genetic alterations and prognostic implications. Histopathology 2016;69:45-53.

20. Sheu SY, Schwertheim S, Worm K, Grabellus F, Schmid KW. Diffuse sclerosing variant of papillary thyroid carcinoma: lack of BRAF mutation but occurrence of RET/PTC rearrangements. Mod Pathol 2007;20:779-87.

21. Li C, Lee KC, Schneider EB, Zeiger MA. BRAF V600E mutation and its association with clinicopathological features of papillary thyroid cancer: a meta-analysis. J Clin Endocrinol Metab 2012;97:4559-70. 